\title{
DETERMINAÇÃO DA DISTÂNCIA ENTRE ALVOS DE MONITORAMENTO UTILIZANDO MODELOS MATEMÁTICOS DISTINTOS E ESTAÇÕES TOTAIS COM DIFERENTES PRECISÕES NOMINAIS
}

\author{
DETERMINATION OF THE DISTANCE BETWEEN MONITORING TARGETS USING DIFFERENT MATHEMATICAL MODELS AND TOTAL \\ STATIONS WITH DIFFERENT NOMINAL ACCURACIES
}

\author{
MARINALVA DE OLIVEIRA LIMA, PEDRO LUIS FAGGION, WANDER DA CRUZ
}

\author{
Universidade Federal do Paraná - UFPR, Campus Politécnico, Programa de Pós-graduação em Ciências Geodésicas. Av. Cel. Francisco \\ H. dos Santos, s/n - Jardim das Américas, Curitiba - PR. Email: nalvalimasr@gmail.com; pedro.faggion@gmail.com; \\ wanderdacruz@gmail.com
}

\begin{abstract}
Resumo - Essa pesquisa teve como objetivo um estudo para a determinação da distância entre alvos de monitoramento, a partir de experimentos com observações coletadas com duas estações totais da marca Leica, de precisões nominais distintas, modelo TCRA1205, 5" angular e $\pm(2 \mathrm{~mm}+2 \mathrm{ppm})$ linear, e a modelo TS15, 1" angular e $\pm(1 \mathrm{~mm}+1,5 \mathrm{ppm})$ linear, e processados utilizando os modelos matemáticos da distância Euclidiana, tradicionalmente utilizada, e a Lei dos Cossenos, esta, com menor número de etapas de cálculo. Para avaliar a acurácia dos dois modelos matemáticos, foram realizadas observações utilizando uma mira horizontal de invar, com a distância entre alvos calibrada utilizando um interferômetro laser, com precisão micrométrica, como padrão de referência para determinar a distância entre os alvos. A fase seguinte da pesquisa foi aplicar os dois modelos matemáticos com os dados obtidos com a estação total modelo TCRA1205 em duas campanhas de medições realizadas em épocas diferentes na UHE Salto Caxias no Estado do Paraná. Os resultados referentes à estimativa, realizada conforme lei das covariâncias, da qualidade das distâncias entre os pontos ocupados com a estação TS15, apresentou resultados melhores, superior a 50\% em relação à estação total modelo TCRA1205 utilizando as duas modelagens matemáticas. Nos resultados referentes às distâncias entre os alvos da mira calculadas pelas modelagens da distância Euclidiana e pela Lei dos Cossenos, submetidos ao teste de hipóteses, t Student, a um nível de confiabilidade de 90\%, pôde-se constatar que as diferenças encontradas entre as distâncias obtidas e a medida de referência, não passou de $3 \mathrm{~mm}$. A menor média do desvio padrão, obtido considerando as diversas séries de observação com os dois equipamentos foi de 2,19mm a 250 metros da mira e processados com a modelagem da distância Euclidiana. Quando se compara os resultados de acordo a posição angular, o resultado que mais se aproximou da medida de referência ocorreu na posição angular a $90^{\circ}$.

Palavras-chave: Law of Cosines; Euclidean distance; Displacement; Monitoring
\end{abstract}

\begin{abstract}
This research aimed at a study to determine the distance between monitoring targets, from experiments with observations collected with two total stations of the Leica brand, of different nominal precision, model TCRA1205, 5" angular and $\pm(2 \mathrm{~mm}+2 \mathrm{ppm})$ linear, and the TS15 model, 1" angular and $\pm(1 \mathrm{~mm}+1.5 \mathrm{ppm})$ linear, and processed using the mathematical models of the Euclidean distance, traditionally used, and the Cosine Law, which has the least number of calculation steps. In order to evaluate the accuracy of the two mathematical models, observations were made using a horizontal invar sight, with the distance between targets calibrated using a laser interferometer, with micrometric precision, as a reference standard to determine the distance between the targets. The next phase of the research was to apply the two mathematical models with the data obtained with the TCRA1205 total station in two measurement campaigns carried out at different times at the Salto Caxias HPP in the State of Paraná. The results referring to the estimate, performed according to the covariance law, of the quality of the distances between the points occupied with the TS15 station, presented better results, higher than 50\% in relation to the total station model TCRA1205 using the two mathematical models. In the results referring to the distances between the targets of the crosshairs calculated by the modeling of the Euclidean distance and by the Law of Cosines, submitted to the test of hypotheses, $t$ Student, at a level of reliability of $90 \%$, it was possible to verify that the differences found between the obtained distances and the reference measurement, did not exceed $3 \mathrm{~mm}$. The lowest average of the standard deviation, obtained considering the various observation series with the two devices, was $2.19 \mathrm{~mm}$ at 250 meters from the crosshairs and processed using the Euclidean distance modeling. When the results are compared according to the angular position, the result that came closest to the reference measurement occurred in the $90^{\circ}$ angular position.
\end{abstract}

Keywords: Law of Cosines; Euclidean distance; Displacement; Monitoring 


\section{INTRODUÇÃO}

Estruturas, sejam elas naturais ou antrópicas, sofrem deformações ao longo de sua vida, e dependendo da sua magnitude, precisam ser monitoradas. Este monitoramento pode ser realizado de forma direta sobre o objeto estudado utilizando equipamentos geotécnicos como, por exemplo, os extensômetros, e/ou por técnicas geodésicas clássicas de levantamentos topográficos até as mais modernas, como escaneamento terrestre e Sistema Global de Navegação por Satélite (GNSS), como apresentado nos estudos de Ibrahim et al. (2010); Pereira (2012) e Graça (2014). Independentemente do método, a coleta de observações pode ser realizada de forma contínua ou periódica, o que está ligado diretamente à dimensão da estrutura, seu comportamento e recursos disponíveis.

As barragens, estruturas antrópicas e de grande porte, sejam elas para geração de energia, acúmulo de resíduos ou captação de água e etc., são exemplos de estruturas que necessitam de constante monitoramento, como apresentado nas pesquisas desenvolvidas por Yigit et al. (2014) na barragem de Ermenek, a segunda barragem mais alta da Turquia; na Usina Hidrelétrica de Mauá, no Rio Tibagi no Paraná por Muguio et al. (2012) e Mezine (2015).

Tendo em vista que os equipamentos utilizados no monitoramento de estruturas têm precisões submilimétrica, estudos têm sido desenvolvidos em busca de metodologias para a obtenção de resultados acurados, e neste sentido, Faggion et al. (2013), em laboratório, utilizou um interferômetro Laser como padrão de referência para testar uma estação total com precisão angular de 5" e linear de $\pm(2 \mathrm{~mm}+2 \mathrm{ppm})$, na determinação de deslocamentos relativos entre pontos. O modelo matemático utilizado pelos autores, para o processamento, foi a Lei dos Cossenos. Os resultados obtidos, demostraram que o modelo matemático utilizado pode ser aplicado para a determinação de distâncias relativa entre pontos com acurácia melhor que um milímetro.

O objetivo deste trabalho é avaliar a distância entre alvos situados em estruturas antrópicas, para fins de monitoramento, a parti de dados coletados com equipamentos de precisões nominal diferentes e processados utilizando dois modelos matemáticos distintos, a distância euclidiana e a Lei dos Cossenos.

\section{MATERIAIS E MÉTODOS}

Para alcançar os objetivos desta pesquisa, foram realizados experimentos simulando alvos dispostos no mesmo plano horizontal utilizando como referência uma mira horizontal de ínvar com distância entre os alvos de 2 metros, no Centro Politécnico, campus III, da Universidade Federal do Paraná (UFPR), e dados de duas campanhas de monitoramento da Usina Hidrelétrica Salto Caxias (UHE Governador José Richa). No experimento realizado na UFPR utilizou-se as estações totais TCRA1205 e TS15, enquanto que na UHE Salto Caxias utilizou-se apenas a estação total TCRA1205. Os dados de ambos os experimentos foram processados utilizando os dois modelos matemáticos, a Lei dos Cossenos e a distância Euclidiana (Fig. 1).

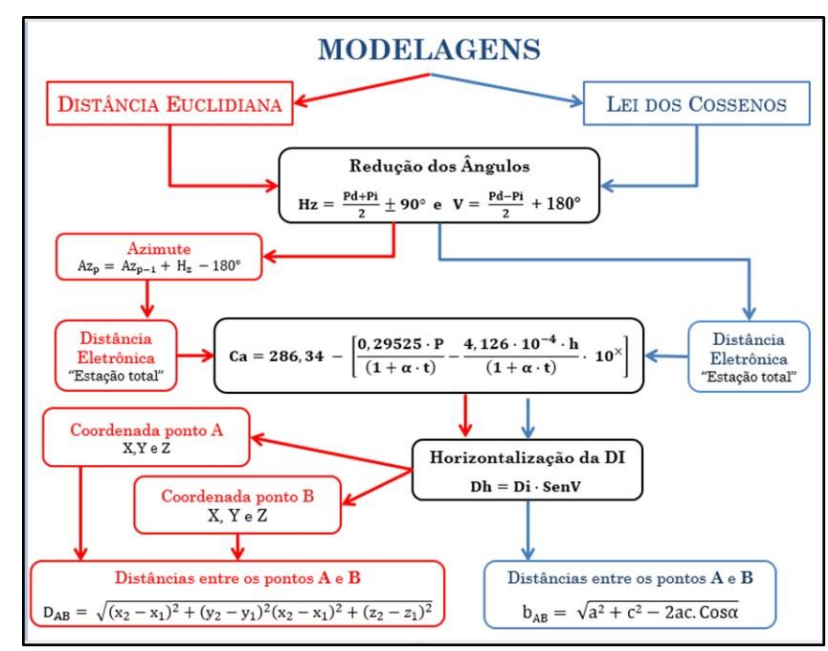

Figura 1 - Etapas de cálculos das modelagens. 


\section{1. Áreas de estudos}

\subsubsection{Usina Hidrelétrica Governador José Richa}

A Usina Hidrelétrica Governador José Richa, mais conhecida como Usina Hidrelétrica de Salto Caxias, localiza-se entre os municípios de Capitão Leônidas Marques e Nova Prata do Iguaçu a aproximadamente $600 \mathrm{~km}$ da capital paranaense, Curitiba (Fig. 2). A barragem é a 8a maior do mundo feita de Concreto Compactado a Rolo, com $912 \mathrm{mil} \mathrm{m}^{3}$ de concreto.

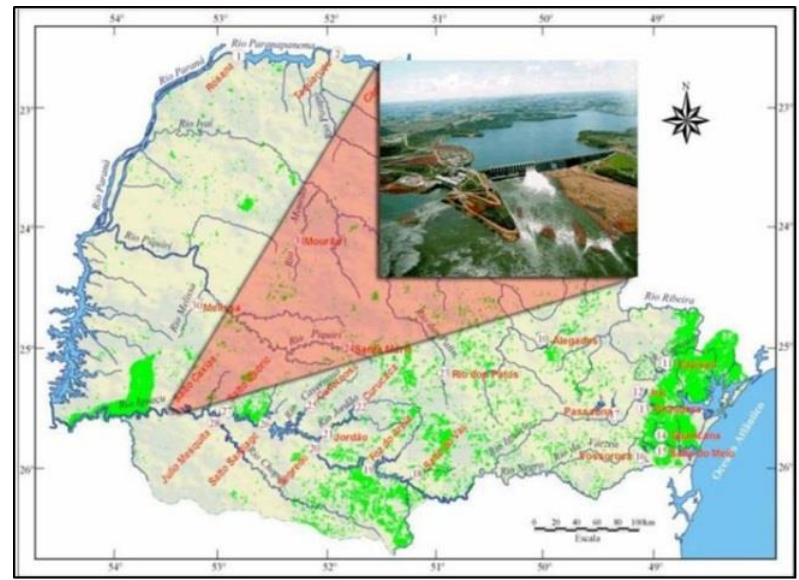

Figura 2- Localização da UHE Salto Caxias (Zocolotti Filho, 2005).
Na área de estudos existem 23 alvos de monitoramento (Marco Geodésico - MGE) fixados no paramento a jusante da barragem. Desses, 7 alvos estão engastados no bloco 5 , 8 alvos engastados no bloco 8 e mais 8 alvos no bloco 11 (Fig. 3).

A determinação da distância entre os alvos, quando da realização das campanhas de medições, são obtidas utilizando a equação de distância Euclidiana, ou seja, a partir das coordenadas dos alvos.

Neste trabalho foram processados os dados dos alvos dos blocos 8 e 11, tendo em vista que estes contemplam um conjunto maior de dados nas campanhas realizadas em novembro de 2016 e julho de 2017.

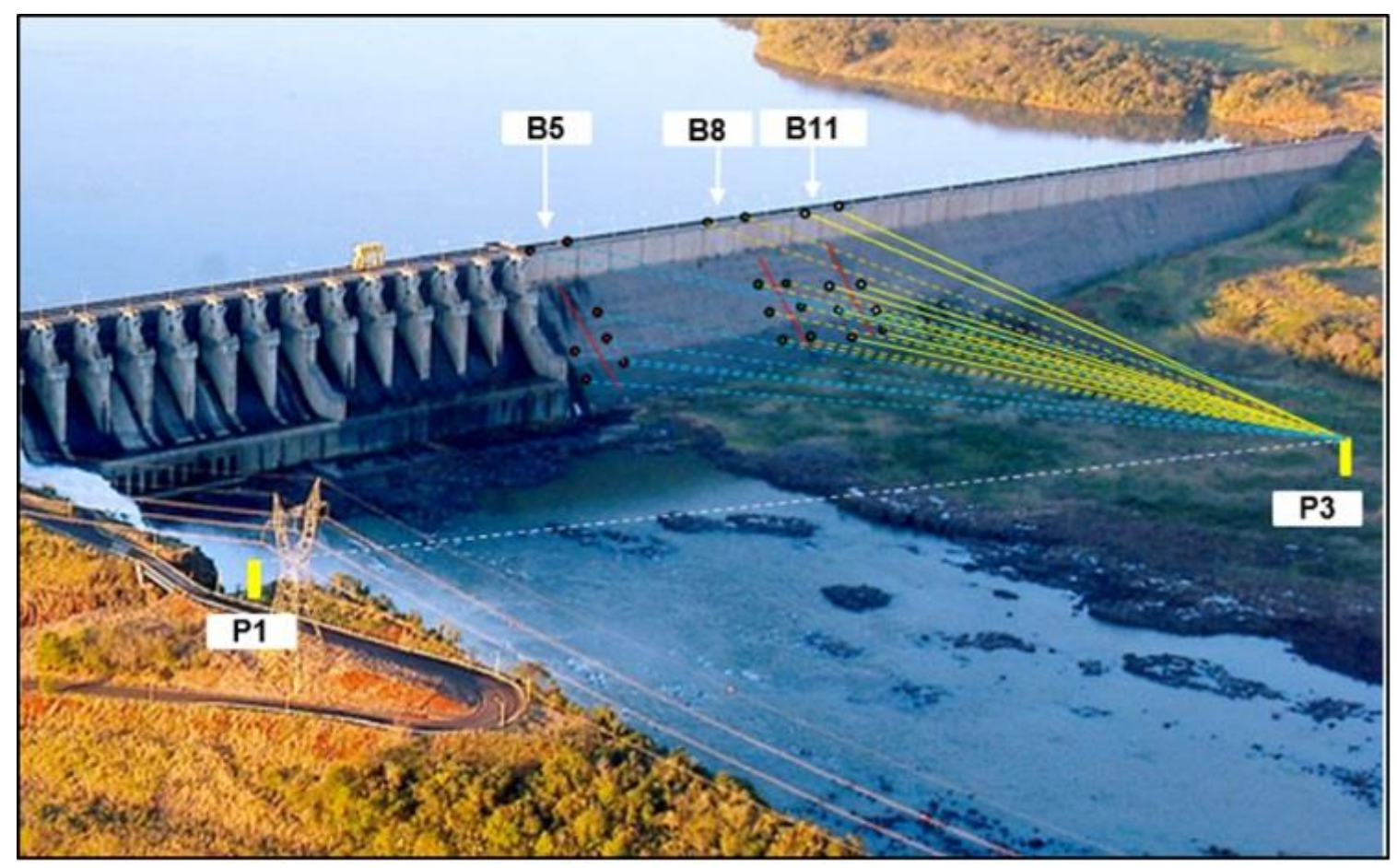

Figura 3 - Campanhas e métodos (Adaptado de COPEL, 2017). 


\subsubsection{Centro Politécnico da UFPR}

O Centro Politécnico é o campus III da UFPR, construído no ano de 1961, sendo o maior campus da UFPR, abrigando diversos cursos de graduação e pós-graduação em diversas áreas de conhecimento. No local foram simuladas distâncias compatíveis com as distâncias entre os alvos e o pilar 3 (P3), na UHE Salto Caxias. Nos experimentos realizados nesta área, utilizou-se como padrão de referência uma mira horizontal de invar (Fig. 4) com o afastamento entre os alvos de dois metros de devidamente calibrada, utilizando o interferômetro laser no

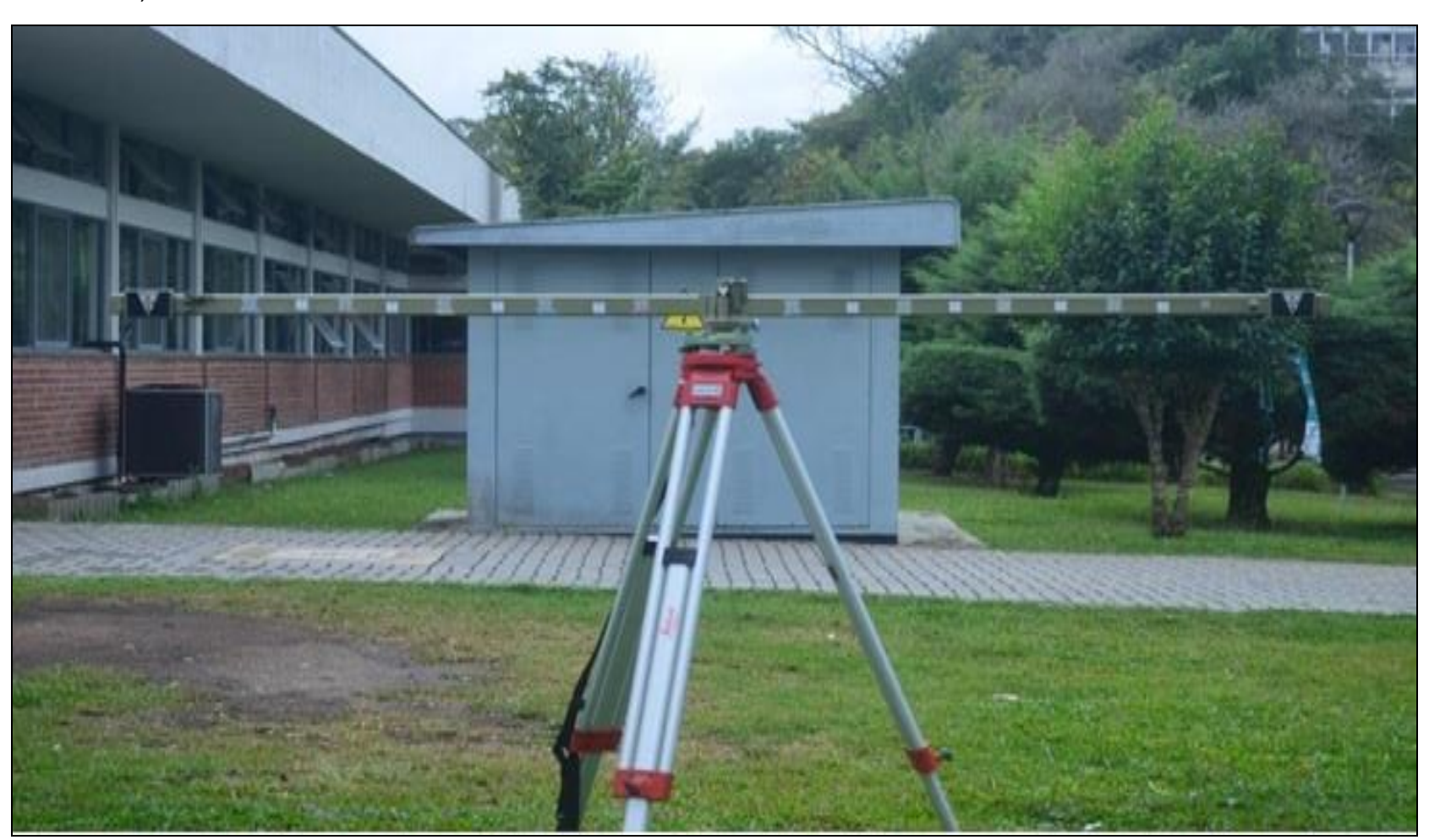

Figura 4 - Mira horizontal de Ínvar de 2 metros (A Autora, 2018).

\subsubsection{Experimentos}

No experimento de campo simulado foram coletados dados utilizando as estações totais da marca Leica modelos TCRA1205 com precisão angular de $5^{\prime \prime}$ e linear de $\pm(2 \mathrm{~mm}+$ $2 \mathrm{ppm})$ e TS15 com precisão angular de 1 " e linear de $\pm(1 \mathrm{~mm}+1,5 \mathrm{ppm})$ utilizando a portadora laser, ambas são classificadas de alta precisão conforme apresentado na Fig. 5. laboratório de instrumentação geodésica (LAIG) da UFPR. A mira de invar possui baixo coeficiente de dilatação, qualidade fundamental para a obtenção da distância com precisão.

Foram coletadas três séries de observações de direções horizontais, ângulos zenitais e distâncias inclinadas, em pares conjugados, utilizando o modo manual de operação das estações totais, mirando os alvos dos extremos da mira horizontal. 
monitoramento da UHE Salto Caxias, processados, em estado bruto.

$\mathrm{Na}$ Fig. 6 apresenta-se um memorando com as informações sobre as campanhas de monitoramento da UHE Salto Caxias e os métodos de processamento para a determinação das distâncias entre os alvos.

Os dados foram observados em pontaria direta (PD) e pontaria inversa (PI) da luneta, em concordância com a NBR 13133/94.

\begin{tabular}{|c|c|}
\hline Épocas e métodos & Dados comparados \\
\hline Distância Euclidiana (D.E) & Novembro/16 x Julho/17 \\
\hline Lei dos Cossenos (L.C) & Novembro/16 x Julho/17 \\
\hline
\end{tabular}

Figura 6 - Campanhas e métodos.

\subsection{Coleta de dados}

Neste experimento, com a mira horizontal de invar posicionada na horizontal, rotacionou-se a mesma, em relação ao alinhamento com a estação total, ocupando três posições distintas, simulando uma situação que se aproximasse das mesmas condições dos alvos engastados nos blocos 5, 8 e 11 no paramento a jusante da barragem. O primeiro passo para criar um cenário similar foi calcular, a partir dos dados brutos do monitoramento da barragem, as distâncias e os ângulos entre os alvos monitorados e o pilar 3, conforme ilustrado nas Figs. 7 e 8.

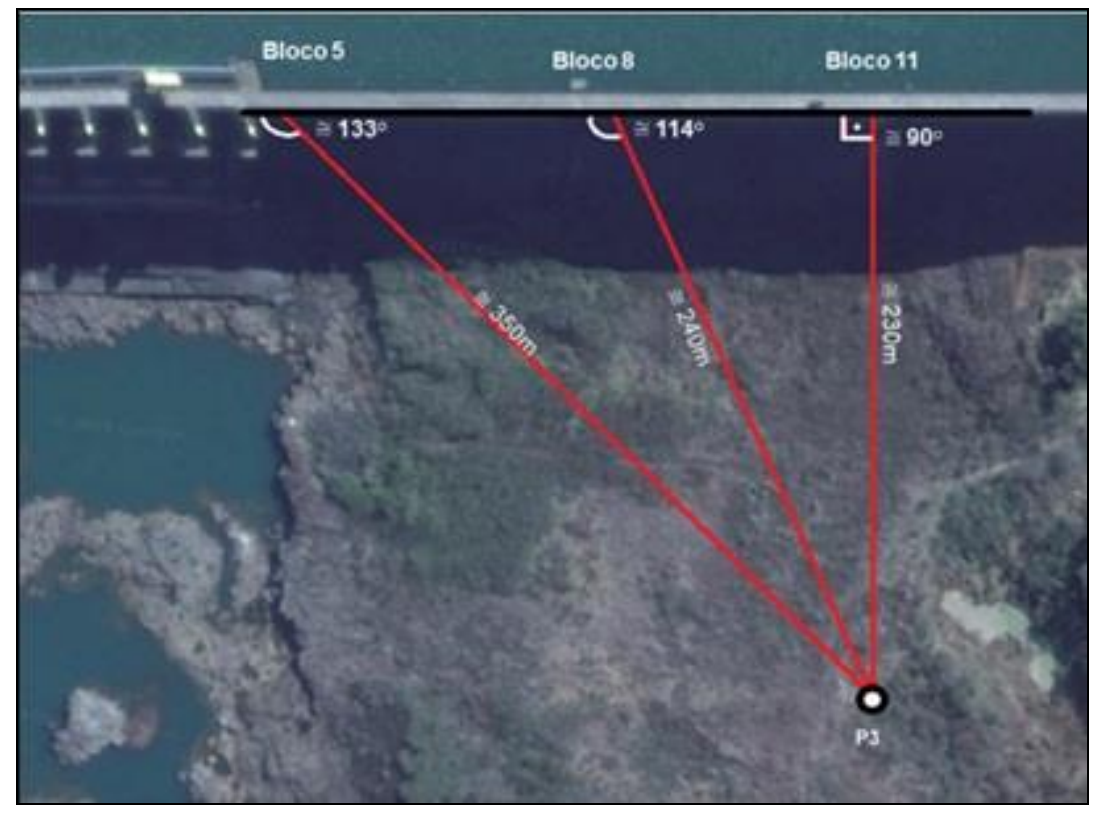

Figura 7 - Blocos monitorados e pilar 3 (Adaptado de Google Earth, 2018).

\begin{tabular}{|c|c|c|}
\hline Blocos & Distância aproximada (m) & Ângulo aproximado com o P3 \\
\hline 5 & 350 & $133^{\circ}$ \\
\hline 8 & 240 & $114^{\circ}$ \\
\hline 11 & 230 & $90^{\circ}$ \\
\hline
\end{tabular}

Figura 8 - Caracterização da localização e distribuição dos alvos (A autora, 2018).

A partir dos dados da figura 8, reproduziu-se um cenário semelhante, dentro do Centro Politécnico, onde a mira horizontal de invar é a referência de distância entre dois alvos simulando os alvos instalados em cada bloco do paramento da barragem a jusante.
A distância entre a estação total e o centro da mira, representa a distância entre os alvos e o pilar 3, que, conforme figuras 9 e 9A, é representado pelas estações E250, E300, E350, locais de ocupação das estações totais para a coleta das observações. 

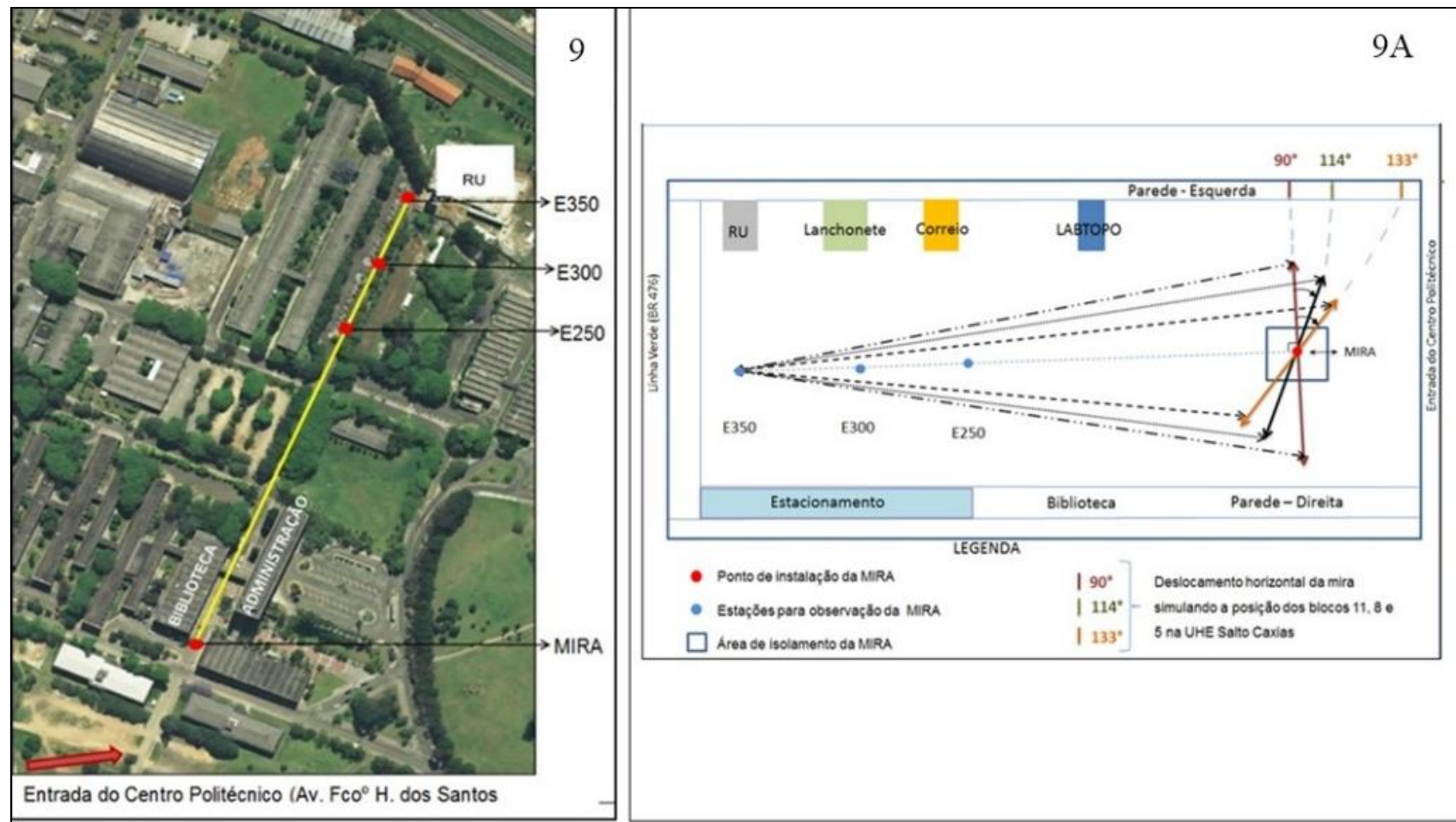

Figuras 9 e 9A - Simulação e croqui dos Blocos monitorados e o pilar 3(Adaptado de Google Earth/Autora, 2018).

Para representar a inclinação das visadas, com relação aos alvos de monitoramento da barragem, sendo o bloco 11 , representado pelo ângulo $90^{\circ}$, o bloco 8 pelo ângulo 114 o e o bloco 5 pelo ângulo 133으, rotacionou-se a mira, com relação ao seu centro, do mesmo ângulo, (Figura 9A).

No experimento do Centro Politécnico, na materialização das estações, procurou-se simular as mesmas condições que foram encontradas UHE Salto Caxias. O procedimento ocorreu na seguinte ordem:

$1^{\circ}$ : Instalou-se a estação total modelo TCAR1205 no ponto denominado MIRA (Figura 9);

$2^{\circ}$ : Instalou-se um prisma com bastão e bipé materializando um ponto a uma distância de 350 metros da estação total instalada no ponto MIRA;

$3^{\circ}$ : Rotacionou-se a estação total no sentido horário até se obter o ângulo de $90^{\circ}$, onde foi materializado o que seria a posição dos alvos do bloco 11; $4^{\circ}$ : A partir do ângulo $90^{\circ}$ a estação total foi girada até se obter o ângulo de $114^{\circ}$, materializando a posição dos alvos do bloco 8 ;

$5^{\circ}$ : Girou-se a estação total, a partir do angulo de $114^{\circ}$ até se obter o ângulo de $133^{\circ} \mathrm{e}$ finalmente materializou-se a posição dos alvos do bloco 5;

$6^{\circ}$ : Orientou-se a estação total novamente no prisma instalado no ponto a $350 \mathrm{~m}$, denominado de E350, de onde com o auxílio de outro prisma instalado em bastão bipé, e mantendo o alinhamento entre o ponto MIRA e a estação E350, o prisma foi deslocado até uma distância de 50m, materializando a estação E300. O mesmo procedimento foi realizado para materializar a estação E250;

$7^{\circ}$ : Retirou-se a estação total mantendo o tripé no ponto MIRA e Instalou-se a mira horizontal de invar;

8: Instalou-se nas estações E350, E300 e E250 tripés com bases nivelante, simulando um sistema de centragem forçada; 
$9^{\circ}$ : Instalou-se o prisma na estação E300 para orientação das estações totais e controle da poligonal;

$10^{\circ}$ : Iniciou-se as observações na estação E350, com a mira horizontal na posição angular de $90^{\circ}$, simulando os alvos do bloco 11, utilizando a estação total TCRA1205 e na sequência a TS15 e assim até o final das observações no ponto a 350 m;

11: Ao final das observações na E350 as estações totais foram transferidas para ocupar a estação E300 e orientadas na E350. Finalizadas as observações com as duas estações totais no ponto ocupado, as mesmas foram transferidas para ocupar a estação E250;

12: A estação E250 foi ocupada com os equipamentos e os mesmos orientados na E350. O processo foi repetido para a estação TS15, finalizando as leituras com a mira horizontal na posição angular de $90^{\circ}$;

\section{RESULTADOS}

\subsection{Simulação com a mira horizontal de invar na horizontal}

Os resultados apresentados na Fig. 11, são referentes a estimativa da qualidade das distâncias entre os pontos ocupados (E250, E300, E350) com as estações totais e o ponto MIRA. A estimativa foi realizada conforme Lei de propagação de covariâncias considerandose as precisões nominais angular e linear das estações totais, a partir de um conjunto (PDPI) de observação em cada ponto de ocupação.Nota-se no demonstrativo na Fig. 12, que ambas as estações totais obtiveram melhores resultados pela Lei dos Cossenos, uma vez que a modelagem envolve um número menor de cálculos e determinação de grandezas auxiliares. Comparando os dois equipamentos, com a estação total TS15 os resultados são acima de 50\% melhores em relação à estação total modelo TCRA1205, utilizando as duas modelagens matemáticas.

Nas Figs. 12 a 15 apresenta-se os resultados referentes às distâncias entre os alvos da mira $13^{\circ}$ : Posteriormente, a mira foi rotacionada para a posição angular de $114^{\circ}$. As observações foram realizadas com as duas estações totais ocupando a estação E250 (orientada na E350) e na sequência foram ocupadas as estações E300 (orientada na E350) e E350 (orientada na E300) e realizada a leitura dos alvos;

$14^{\circ}$ : Finalizando a etapa 13 , a mira foi rotacionada para a posição angular de $133^{\circ} \mathrm{e}$ nesta posição angular foram realizadas as observações com as duas estações totais ocupando as estações E350, E300 e finalizando a coleta de dados do experimento na estação E250, repetindo-se as mesmas orientações da etapa 13.

As posições angulares para as quais a mira horizontal foi colocada estão ilustradas nas figuras 10,10A, 10B, 10C, 10D e 10E.

calculadas pelas modelagens da distância Euclidiana e pela Lei dos Cossenos, utilizando as estações totais TCRA1205 e TS15. Os resultados foram submetidos ao teste de hipóteses, t Student, em que se verifica, a um nível de confiança de $90 \%$, se os valores são iguais estatisticamente ao valor de referência, a distância entre os alvos de mira horizontal de invar que é de 2 metros. As distâncias calculadas foram comparadas estatisticamente a um grau de confiabilidade de $90 \%$ com o valor de referência, onde os resultados mostraram a igualdade estatística das mesmas. Analisando os resultados apresentados nas Figs. 12 e 13, de acordo com a distância da estação total em relação a mira horizontal, observa-se igualdade nas médias das estações 250 e 350. A menor diferença para os dois equipamentos, Figs. 12 e 13, foi obtida na distância de 300 metros da mira. A menor média do desvio padrão, considerando as diversas séries de observação foi de $2,19 \mathrm{~mm}$ e são referentes a estação 250 e a maior para a estação 350, com o valor de $3,50 \mathrm{~mm}$. 


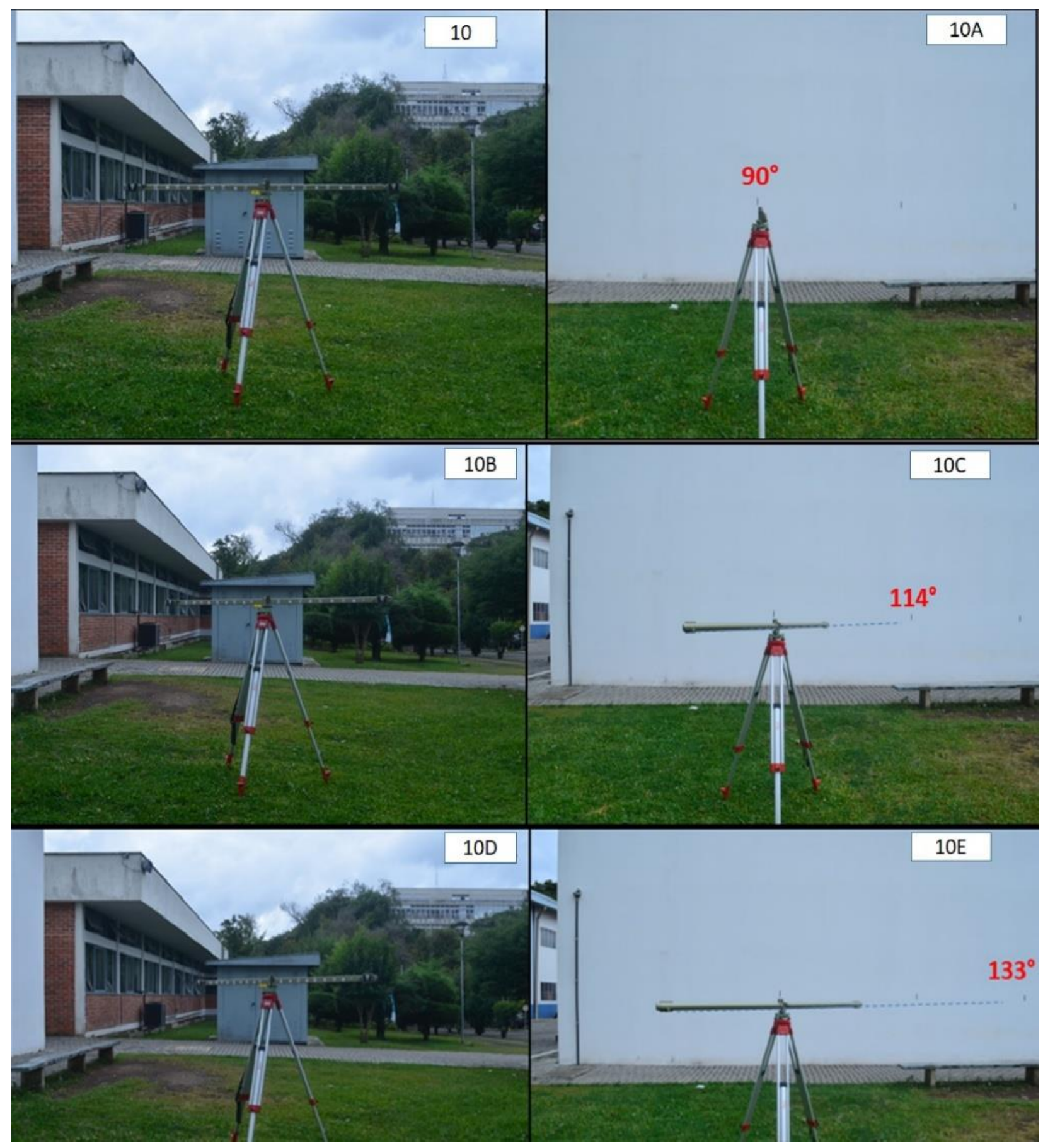

Figura $10(A, B, C, D, E)$ - Posições angulares da mira em $90^{\circ}, 114^{\circ}$ e $133^{\circ}$.

\begin{tabular}{|c|c|c|c|c|}
\hline $\begin{array}{c}\text { Distância entre a } \\
\text { Estação Total e a } \\
\text { Mira horizontal } \\
(\mathrm{m})\end{array}$ & \multicolumn{2}{|c|}{$\begin{array}{c}\text { Desvio padrão Distância Euclidiana } \\
(\mathrm{mm})\end{array}$} & \multicolumn{2}{c|}{ Desvio padrão Lei dos Cossenos (mm) } \\
\cline { 2 - 5 } & TCRA1205 & TS15 & TCRA1205 & TS15 \\
\hline 250,108 & 10,00 & 4,4 & 6,5 & 2,3 \\
\hline 300,060 & 10,00 & 4,4 & 7,8 & 2,5 \\
\hline 350,582 & 10,00 & 4,4 & 8,9 & 2,7 \\
\hline \multicolumn{5}{|c|}{ Médias } \\
\hline 300,250 & 10,00 & 4,4 & 7,33 & 2,5 \\
\hline
\end{tabular}

Figura 11 - Estimativas de acordo com a distância observada.

e as precisões nominais das estações totais. 


\begin{tabular}{|c|c|c|c|c|c|c|c|c|}
\hline \multirow[t]{2}{*}{$\begin{array}{l}\text { Distância da } \\
\text { Estação Total em } \\
\text { relação a Mira } \\
\text { (m) }\end{array}$} & \multirow[t]{2}{*}{$\begin{array}{c}\text { Mira em relação } \\
\text { a linha de visada } \\
\text { da Estação Total } \\
\left(^{\circ}\right)\end{array}$} & \multicolumn{2}{|c|}{$\begin{array}{l}\text { Comprimento } \\
\text { Calculado } \\
(\mathrm{m})\end{array}$} & \multicolumn{2}{|c|}{$\begin{array}{l}\text { Desvio padrão } \\
\text { obtido } \\
\text { (mm) }\end{array}$} & \multicolumn{2}{|c|}{$\begin{array}{c}\text { Diferença com } \\
\text { o valor de } \\
\text { referência } \\
(\mathrm{mm})\end{array}$} & \multirow{2}{*}{$\begin{array}{c}\text { Resultado do } \\
\text { teste Estatístico t } \\
\text { Student a } 90 \% \\
\text { de } \\
\text { confiabilidade }\end{array}$} \\
\hline & & & Média & & Média & & Média & \\
\hline \multirow{3}{*}{250} & $90^{\circ}$ & 1,999 & \multirow{3}{*}{2,001} & 2,24 & \multirow{3}{*}{2,19} & 1 & \multirow{3}{*}{1,7} & Igual \\
\hline & $114^{\circ}$ & 2,003 & & 2,11 & & 3 & & Igual \\
\hline & $133^{\circ}$ & 2,001 & & 2,21 & & 1 & & Igual \\
\hline \multirow{3}{*}{300} & $90^{\circ}$ & 1,999 & \multirow{3}{*}{2,000} & 2,60 & \multirow{3}{*}{3,16} & 1 & \multirow{3}{*}{1,0} & Igual \\
\hline & $114^{\circ}$ & 2,001 & & 3,47 & & 1 & & Igual \\
\hline & $133^{\circ}$ & 2,001 & & 3,42 & & 1 & & Igual \\
\hline \multirow{3}{*}{350} & $90^{\circ}$ & 1,995 & \multirow{3}{*}{1,997} & 3,47 & \multirow{3}{*}{3,39} & 5 & \multirow{3}{*}{3,0} & Igual \\
\hline & $114^{\circ}$ & 1,999 & & 4,12 & & 1 & & Igual \\
\hline & $133^{\circ}$ & 1,997 & & 2,87 & & 3 & & Igual \\
\hline
\end{tabular}

Figura 22 - Distância Euclidiana - estação total TCRA1205.

\begin{tabular}{|c|c|c|c|c|c|c|c|c|}
\hline $\begin{array}{l}\text { Distância da } \\
\text { Estação Total em } \\
\text { relação a Mira } \\
\text { (m) }\end{array}$ & $\begin{array}{c}\text { Mira em relação } \\
\text { a linha de visada } \\
\text { da Estação Total } \\
\left({ }^{\circ}\right)\end{array}$ & $\begin{array}{l}\text { Compr } \\
\text { Calc }\end{array}$ & $\begin{array}{l}\text { nento } \\
\text { ado }\end{array}$ & $\begin{array}{r}\text { Desvio } \\
\text { ob } \\
(\mathrm{m}\end{array}$ & $\begin{array}{l}\text { adrão } \\
\text { lo } \\
\text { ) }\end{array}$ & $\begin{array}{r}\text { Difere } \\
\text { o va } \\
\text { refe } \\
\quad(n\end{array}$ & $\begin{array}{l}\text { ça com } \\
\text { or de } \\
\text { incia } \\
\text { m) } \\
\text { Média } \\
\end{array}$ & $\begin{array}{c}\text { Resultado do } \\
\text { teste Estatístico t } \\
\text { Student a } 90 \% \\
\text { de } \\
\text { confiabilidade }\end{array}$ \\
\hline \multirow{3}{*}{250} & $90^{\circ}$ & 1,999 & \multirow{3}{*}{2,001} & 2,24 & \multirow{3}{*}{2,19} & 1 & \multirow{3}{*}{1,7} & Igual \\
\hline & $114^{\circ}$ & 2,003 & & 2,11 & & 3 & & Igual \\
\hline & $133^{\circ}$ & 2,001 & & 2,21 & & 1 & & Igual \\
\hline \multirow{3}{*}{300} & $90^{\circ}$ & 1,999 & \multirow{3}{*}{1,999} & 2,60 & \multirow{3}{*}{3,30} & 1 & \multirow{3}{*}{1,3} & Igual \\
\hline & $114^{\circ}$ & 1,998 & & 3,89 & & 2 & & Igual \\
\hline & $133^{\circ}$ & 2,001 & & 3,42 & & 1 & & Igual \\
\hline \multirow{3}{*}{350} & $90^{\circ}$ & 1,995 & \multirow{3}{*}{1,997} & 3,50 & \multirow{3}{*}{3,50} & 5 & \multirow{3}{*}{3,0} & Igual \\
\hline & $114^{\circ}$ & 1,999 & & 4,12 & & 1 & & Igual \\
\hline & $133^{\circ}$ & 1,997 & & 2,87 & & 3 & & Igual \\
\hline
\end{tabular}

Figura 33 - Distância Euclidiana - estação total TS15.

Quando se compara os resultados de acordo a posição angular, o resultado que mais se aproximou do comprimento de referência, foi obtido na posição a $90^{\circ}$ e $133^{\circ}$ a 250 e a 300 metros da mira, com diferença de apenas $0,001 \mathrm{~mm}$. Nas mesmas posições angulares, 0 resultado com a maior diferença está na posição de $114^{\circ}$. Observa-se que a 350 metros da mira os valores obtidos com os dois equipamentos somente foi divergente na média do desvio padrão obtido.

Os resultados obtidos pela modelagem da Lei dos Cossenos estão apresentados nas Figs. 14 e 15 a seguir.
Analisando-se os resultados obtidos com o equipamento estação total TCRA1205, menor variação da distância com relação ao valor de referência foi na estação 300, com média de $0,30 \mathrm{~mm}$. O comprimento calculado que mais se aproximou do comprimento da mira ocorreu na estação 300 com os dois equipamentos utilizados no levantamento, com média de 2 metros.

Na Fig. 15, apresenta-se os resultados obtidos com o equipamento TS15. Na estação a 250 metros da mira, verificou-se o menor desvio padrão, com média de 2,5mm. A 350 metros ocorreu o comprimento que menos se aproximou da medida de referência. 


\begin{tabular}{|c|c|c|c|c|c|c|c|c|}
\hline \multirow[t]{2}{*}{$\begin{array}{l}\text { Distância da } \\
\text { Estação Total em } \\
\text { relação a Mira } \\
\text { (m) }\end{array}$} & \multirow[t]{2}{*}{$\begin{array}{c}\text { Mira em relação } \\
\text { a linha de visada } \\
\text { da Estação Total } \\
\left(^{\circ}\right)\end{array}$} & \multicolumn{2}{|c|}{$\begin{array}{l}\text { Comprimento } \\
\text { Calculado } \\
\text { (m) }\end{array}$} & \multicolumn{2}{|c|}{$\begin{array}{c}\text { Desvio padrão } \\
\text { obtido } \\
(\mathrm{mm})\end{array}$} & \multicolumn{2}{|c|}{$\begin{array}{c}\text { Diferença com } \\
\text { o valor de } \\
\text { referência } \\
(\mathrm{mm})\end{array}$} & \multirow{2}{*}{$\begin{array}{c}\text { Resultado do } \\
\text { teste Estatístico t } \\
\text { Student a } 90 \% \\
\text { de } \\
\text { confiabilidade }\end{array}$} \\
\hline & & & Média & & Média & & Média & \\
\hline \multirow{3}{*}{250} & $90^{\circ}$ & 1,999 & \multirow{3}{*}{2,001} & 3,01 & \multirow{3}{*}{3,18} & 1 & \multirow{3}{*}{1,7} & Igual \\
\hline & $114^{\circ}$ & 2,001 & & 4,15 & & 1 & & Igual \\
\hline & $133^{\circ}$ & 2,003 & & 2,39 & & 3 & & Igual \\
\hline \multirow{3}{*}{300} & $90^{\circ}$ & 2,001 & \multirow{3}{*}{2,000} & 2,99 & \multirow{3}{*}{2,92} & 1 & \multirow{3}{*}{0,3} & Igual \\
\hline & $114^{\circ}$ & 2,000 & & 3,14 & & 0 & & Igual \\
\hline & $133^{\circ}$ & 2,000 & & 2,63 & & 0 & & Igual \\
\hline \multirow{3}{*}{350} & $90^{\circ}$ & 1,999 & \multirow{3}{*}{1,998} & 5,64 & \multirow{3}{*}{5,04} & 1 & \multirow{3}{*}{2,0} & Igual \\
\hline & $114^{\circ}$ & 1,998 & & 4,34 & & 2 & & Igual \\
\hline & $133^{\circ}$ & 1,997 & & 5,13 & & 3 & & Igual \\
\hline
\end{tabular}

Figura 44 - Lei dos Cossenos - estação total TCRA1205.

\begin{tabular}{|c|c|c|c|c|c|c|c|c|}
\hline \multirow[t]{2}{*}{$\begin{array}{l}\text { Distância da } \\
\text { Estação Total em } \\
\text { relação a Mira } \\
\text { (m) }\end{array}$} & \multirow[t]{2}{*}{$\begin{array}{c}\text { Mira em relação } \\
\text { a linha de visada } \\
\text { da Estação Total } \\
\left({ }^{\circ}\right)\end{array}$} & \multicolumn{2}{|c|}{$\begin{array}{l}\text { Comprimento } \\
\text { Calculado } \\
\text { (m) }\end{array}$} & \multicolumn{2}{|c|}{$\begin{array}{c}\text { Desvio padrão } \\
\text { obtido } \\
(\mathrm{mm})\end{array}$} & \multicolumn{2}{|c|}{$\begin{array}{c}\text { Diferença com } \\
\text { o valor de } \\
\text { referência } \\
(\mathrm{mm})\end{array}$} & \multirow{2}{*}{$\begin{array}{c}\text { Resultado do } \\
\text { teste Estatístico t } \\
\text { Student a } 90 \% \\
\text { de } \\
\text { confiabilidade }\end{array}$} \\
\hline & & & Média & & Média & & Média & \\
\hline \multirow{3}{*}{250} & $90^{\circ}$ & 1,999 & \multirow{3}{*}{2,001} & 2,90 & \multirow{3}{*}{2,50} & 1 & \multirow{3}{*}{1,7} & Igual \\
\hline & $114^{\circ}$ & 2,003 & & 2,47 & & 3 & & Igual \\
\hline & $133^{\circ}$ & 2,001 & & 2,14 & & 1 & & Igual \\
\hline \multirow{3}{*}{300} & $90^{\circ}$ & 1,999 & \multirow{3}{*}{2,000} & 2,59 & \multirow{3}{*}{3,36} & 1 & \multirow{3}{*}{1,7} & Igual \\
\hline & $114^{\circ}$ & 1,998 & & 3,88 & & 2 & & Igual \\
\hline & $133^{\circ}$ & 2,002 & & 3,62 & & 2 & & Igual \\
\hline \multirow{3}{*}{350} & $90^{\circ}$ & 1,995 & \multirow{3}{*}{1,997} & 3,81 & \multirow{3}{*}{3,33} & 5 & \multirow{3}{*}{2,7} & Igual \\
\hline & $114^{\circ}$ & 1,999 & & 3,74 & & 1 & & Igual \\
\hline & $133^{\circ}$ & 1,998 & & 2,44 & & 2 & & Igual \\
\hline
\end{tabular}

Figura 55 - Lei dos Cossenos - estação total TS15.

\subsection{Dados da barragem}

Os resultados obtidos a partir dos dados brutos das campanhas de monitoramento da UHE Salto Caxias foram processados conforme apresentado na Fig. 6 no tópico 2.2. A distância entre os alvos e o ponto de observação, P3 foi de aproximadamente $240 \mathrm{~m}$, conforme ilustrado nas Figs. 7 e 8. Adotou-se como medida de referência a distância obtida com a modelagem da distância Euclidiana, tendo em vista que este método é o mais usado para esta finalidade.

\subsection{Campanha de novembro de 2016}

A Fig. 16 mostra os resultados referentes aos alvos engastados nos blocos 8 e 11 para análise entre os dois métodos na mesma campanha de medição.

Analisando os resultados, nota-se que os valores da distância entre os alvos 21-22, 2324 e 29-30 não são estatisticamente iguais, podendo ser justificado pelo valor do desvio padrão obtido, pois o mesmo apresenta um valor inferior ao valor da diferença entre as distâncias de referência, distância Euclidiana. A média da diferença entre as duas modelagens é de $1,9 \mathrm{~mm}$. 


\begin{tabular}{|c|c|c|c|c|c|}
\hline \multirow[t]{2}{*}{ Alvos } & \multirow{2}{*}{$\begin{array}{c}\text { Distância } \\
\text { "Referência" } \\
\text { EUCLIDIANA (D.E) } \\
\text { Nov/16 } \\
\text { (m) }\end{array}$} & \multirow{2}{*}{$\begin{array}{c}\text { Distância } \\
\text { "Referência" LEI } \\
\text { DOS COSSENOS } \\
\text { (L.C) Nov/16 } \\
\text { (m) }\end{array}$} & \multirow{2}{*}{$\begin{array}{l}\text { Desvio Padrão } \\
\text { obtido L.C } \\
(\mathrm{mm})\end{array}$} & \multirow{2}{*}{$\begin{array}{c}\text { Diferença } \\
\text { D.E-L.C } \\
(\mathrm{mm})\end{array}$} & $\begin{array}{c}\text { Resultado do teste Estatístico } \\
\text { t Student a } 90 \% \text { de } \\
\text { CONFIABILIDADE }\end{array}$ \\
\hline & & & & & LEI DOS COSSENOS \\
\hline $19-20$ & 2,820 & 2,820 & 1 & 0 & Igual \\
\hline $21-22$ & 1,822 & 1,816 & 1 & 6 & Diferente \\
\hline $23-24$ & 1,714 & 1,717 & 1 & 3 & Diferente \\
\hline $29-30$ & 1,314 & 1,313 & 0 & 1 & Diferente \\
\hline $31-32$ & 1,511 & 1,510 & 0 & 1 & Igual \\
\hline $33-34$ & 1,280 & 1,279 & 0 & 1 & Igual \\
\hline $35-36$ & 9,504 & 9,503 & 1 & 1 & Igual \\
\hline Média & 2,852 & 2,851 & 0,6 & 1,9 & \\
\hline
\end{tabular}

Figura 16 - Resultados: Distância Euclidiana x Lei dos Cossenos - Nov/16.

\subsection{Campanha de julho 2017}

Na Fig. 17 comparando os resultados obtidos pelas duas modelagens na campanha realizada em julho de 2017, observa-se que as distâncias calculadas pela Lei dos Cossenos são estatisticamente iguais com relação aos resultados calculados pela distância Euclidiana. A maior diferença entre as modelagens estudadas é de $1 \mathrm{~mm}$ e se refere a distância entre os alvos 35-36, localizados no bloco 11.

\begin{tabular}{|c|c|c|c|c|c|}
\hline \multirow[t]{2}{*}{ Alvos } & \multirow{2}{*}{$\begin{array}{c}\text { Distância } \\
\text { "Referência" } \\
\text { EUCLIDIANA } \\
\text { (D.E) Jul/17 } \\
\text { (m) }\end{array}$} & \multirow{2}{*}{$\begin{array}{l}\text { Distância } \\
\text { "Referência" } \\
\text { LEI DOS } \\
\text { COSSENOS } \\
\text { (L.C) Jul/17 } \\
\text { (m) }\end{array}$} & \multirow{2}{*}{$\begin{array}{c}\text { Desvio Padrão } \\
\text { obtido L.C } \\
(\mathrm{mm})\end{array}$} & \multirow{2}{*}{$\begin{array}{c}\text { Diferença } \\
\text { D.E-L.C } \\
(\mathrm{mm})\end{array}$} & $\begin{array}{c}\text { Resultado do teste Estatístico } \\
\text { t Student a 90\% de } \\
\text { CONFIABILIDADE }\end{array}$ \\
\hline & & & & & LEI DOS COSSENOS \\
\hline $19-20$ & 2,815 & 2,815 & 1 & 0 & Igual \\
\hline $21-22$ & 1,815 & 1,815 & 0 & 0 & Igual \\
\hline $23-24$ & 1,717 & 1,717 & 1 & 0 & Igual \\
\hline $29-30$ & 1,306 & 1,306 & 1 & 0 & Igual \\
\hline $31-32$ & 1,495 & 1,495 & 2 & 0 & Igual \\
\hline $33-34$ & 1,282 & 1,282 & 1 & 0 & Igual \\
\hline $35-36$ & 9,508 & 9,507 & 1 & 1 & Igual \\
\hline Média & 2,848 & 2,848 & 1 & 0,1 & \\
\hline
\end{tabular}

\section{CONCLUSÕES}

Os resultados dos experimentos simulados observados em campo utilizando as duas estações totais e processados a partir das modelagens da distância Euclidiana e da Lei dos Cossenos na determinação da distância entre os alvos da mira horizontal de ínvar, são estatisticamente iguais ao Padrão de Referência utilizado, obtido através da calibração da distância entre os alvos da mira horizontal de invar no Laboratório de Instrumentação Geodésica da UFPR.

A média da diferença entre as distâncias medidas e comparadas não passou de $3 \mathrm{~mm}$; comparando os equipamentos, a média do desvio padrão obtido com as duas estações totais TCRA1205 e TS15 foi de 2,19mm a 250 metros da mira com a modelagem da distância Euclidiana, a 350 metros com a modelagem da Lei dos Cossenos, a TCRA1205 obteve a maior média do desvio padrão, de $5,04 \mathrm{~mm}$. Ambos os resultados, estão dentro dos valores de desvios padrões estimados para os equipamentos utilizados e dentro de suas precisões nominais de acordo com o fabricante.

$\mathrm{Na}$ comparação dos resultados dos dados brutos do monitoramento da UHE Salto Caxias também processados utilizando as duas modelagens e coletados com a estação total TCRA1205, quando se compara as modelagens na mesma campanha, nos resultados de novembro de 2016, três distâncias são diferentes estatisticamente, onde o desvio padrão das mesmas é menor do que a diferença das distâncias comparadas. A média do desvio padrão foi de 0,6 $\mathrm{mm}$ na campanha de novembro de $2016 \mathrm{e}$ de $1 \mathrm{~mm}$ na campanha de julho de 2017; quando se compara as diferenças entre os 
comprimentos, na campanha de novembro foi de $1,9 \mathrm{~mm}$ e na de julho de $0,1 \mathrm{~mm}$. De acordo com o teste estatístico aplicado, $\mathrm{t}$ Student, as distâncias são iguais estatisticamente na campanha de julho de 2017, a um grau de $90 \%$ de confiabilidade.

De uma forma geral, os resultados dos experimentos realizados no Centro Politécnico, mostraram igualdade estatística para o cálculo da distância pela Lei dos Cossenos e distância Euclidiana, independentemente do equipamento utilizado. Estes resultados mostraram que as duas modelagens podem ser aplicadas na determinação de distâncias entre alvos de monitoramento.

Alisando os resultados das duas campanhas de monitoramento, pode-se concluir que, assim como nos experimentos realizados no centro politécnico, os resultados obtidos com os dados da barragem mostram que a Lei dos Cossenos pode ser usada para monitoramento de grandes estruturas assim como a distância Euclidiana, já amplamente utilizada.

\section{AGRADECIMENTOS}

Agradecemos à CAPES e CNPq pelo apoio à pesquisa e suporte aos estudos do primeiro autor, processo 134640/2016-0.

\section{REFERÊNCIAS}

ALENCAR FILHO, E. 1969. Curso de Trigonometria Plana. São Paulo. Nobel S.A. 9a Ed. 531p.

ASSOCIAÇÃO BRASILEIRA DE NORMAS TÉCNICAS (ABNT). 1994. NBR 13133: Execução de levantamento topográfico. Rio de Janeiro, 35p.

BREED, C. B.; HOSMER, G, L.; BONE A, J. 2012. The Principles And Practice Of Surveying. $614 p$.

COPEL - Companhia Paranaense de Energia. Geração. 2016. Disponível em: http://www.copel.com/hpcopel/root/nivel2.js p?endereco=\%2Fhpcopel\%2Froot\%2Fpagcop el2.nsf\%2F044b34faa7cc1143032570bd0059 aa29\%2F9bdc37f6b8c44b810325741200587d b7. Acesso em 30 Agosto 2017.
DEPARTMENT OF THE ARMY - U. S. ARMY CORPS OF ENGENEERS. 2002. Manual 1110-11004 Deformation Monitoring and Control Surveying. Washington, DC. 102p.

FAGGION, L, P.; VEIGA, A, K.; SILVA, L, F.; HENRIQUE, T.; SILVIO, D, A. 2013. Estudo da aplicação de estações totais para a determinação de deslocamentos relativos em laboratório. Curitiba - Brasil. Boletim de Ciências Geodésicas. ISSN: 1982-2170, sec. Artigos, v. 19, $\mathrm{n}^{\circ} 1$.

GEMAEL, C; MACHADO, L, M, A; WANDRESEN, R. 2015. Introdução ao ajustamento de observações: aplicações geodésicas. Curitiba: Ed. UFPR. 428p.

Google Earth. 2017. Disponível em: <https://w w w w google.com .br/earth/download/ge/agree.html>. Acesso em 28 maio 2018.

GRAÇA, N.L.S.S. 2014. Validação da determinação de deslocamentos relativos em obras de engenharia obtidos com técnica geodésica e medidores triortogonais de junta. Dissertação de Mestrado - Curso de PósGraduação em Ciências Geodésicas. Universidade Federal do Paraná, Curitiba. $106 \mathrm{p}$.

IBRAHIM, M.R., JAFAR, F., YAHYA, Z., SAMAD, A.M, A 2010. Feasibility Study of Building Structural deformation Monitoring Using Global Positioning System (GPS), Terrestrial Surveying Technique (TST) and Crack Gauge Measurement (CGM). 6th International Colloquium on Signal Processing \& Its Applications (CSPA).

LEICA. 2017. Leica TSPS1200+ User Manual. Version 9.1.0. Leica Geosystems AG. Heerbrugg, Switzerland. 228p.

MEZINE, W. A. 2015. Avaliação de métodos de redução de sondagens batimétricas ao nível d'água instantâneo para monitoramento de assoreamento: estudo de caso do reservatório da usina hidrelétrica Mauá. Dissertação de Mestrado - Curso de PósGraduação em Ciências Geodésicas. Universidade Federal do Paraná, Curitiba.

MIKHAIL, E.; ACKERMAN, F. 1976. Observations and Least Squares. University Press of America. 497p. 
MUGUIO, M. R. 2012. Implantação e análise da estação GNSS para o monitoramento contínuo da barragem da Usina Hidrelétrica de Mauá. Dissertação de Mestrado - Curso de Pós-Graduação em Ciências Geodésicas. Universidade Federal do Paraná, Curitiba.

PEREIRA, A. R. A.; SILVA, T. F.; 2012. Monitoramento geodésico de uma encosta em processo de deslizamento de terra; IV Simpósio Brasileiro de Ciências Geodésicas e Tecnologias da Geoinformacao Recife - PE, 06 - 09 de Maio de 2012 p. 001 - 009.

SILVA, I. DA; SEGANTINE, P. C. L. 2015. Topografia Para Engenharia: Teoria e Prática de Geomática. 1. ed. Elsevier. 432p.

ZOCOLOTTI FILHO, C. A. 2005. Utilização de técnicas de poligonação de precisão para o monitoramento de pontos localizados em galerias de inspeção: estudo de caso da U. H. de Salto Caxias. Curso de Pós-Graduação em Ciências Geodésicas. Universidade Federal do
Paraná, Curitiba, Dissertação de Mestrado, $112 p$.

WOLF, P. R. 1980. Adjustment computations: pratical least squares for surveyors. 2.ed. Wisconsin: PBL. 280p.

YIGIT, C. O; ALCAY, S; CEYLAN, A . 2016. Displacement response of a concrete arch dam to seasonal temperature fluctuations and reservoir level rise during the first filling period: evidence from geodetic data. Geomatics, Natural Hazards and Risk, 2016 Vol. 7, No. 4, 1489_1505, http://dx.doi.org/10.1080/19475705.2015.10 47902.
Submetido em 11/09/2019 Aceito em 16/04/2020 